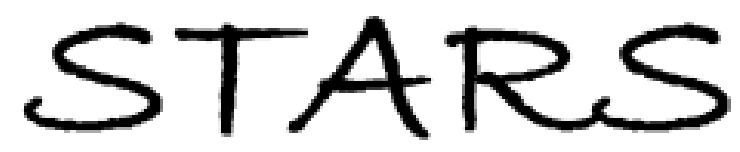

University of Central Florida

STARS

$1-1-2008$

\title{
Free-space optical system performance for laser beam propagation through non-Kolmogorov turbulence
}

Italo Toselli

Larry C. Andrews

University of Central Florida

Ronald L. Phillips

Valter Ferrero

Find similar works at: https://stars.library.ucf.edu/facultybib2000

University of Central Florida Libraries http://library.ucf.edu

This Article; Proceedings Paper is brought to you for free and open access by the Faculty Bibliography at STARS. It has been accepted for inclusion in Faculty Bibliography 2000s by an authorized administrator of STARS. For more information, please contact STARS@ucf.edu.

\section{Recommended Citation}

Toselli, Italo; Andrews, Larry C.; Phillips, Ronald L.; and Ferrero, Valter, "Free-space optical system performance for laser beam propagation through non-Kolmogorov turbulence" (2008). Faculty Bibliography 2000s. 1064.

https://stars.library.ucf.edu/facultybib2000/1064

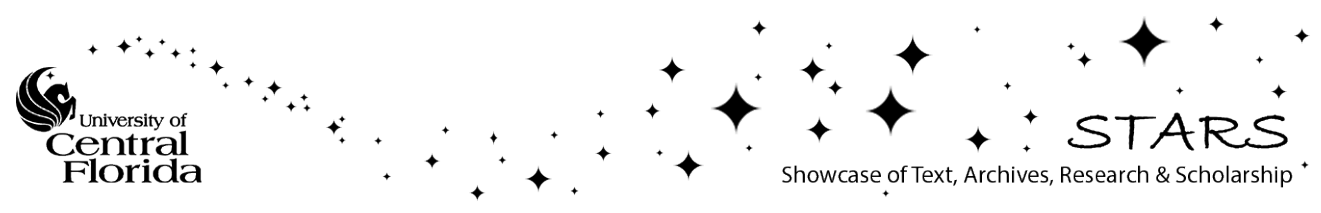




\section{Free-space optical system performance for laser beam propagation through non-Kolmogorov turbulence}

\author{
Italo Toselli \\ Politecnico di Torino \\ Optical Communications Group \\ 10128 Turin, Italy
}

\section{Larry C. Andrews}

University of Central Florida

Department of Mathematics

Orlando, Florida 32816

\section{Ronald L. Phillips}

Florida Space Institute

Mail Stop: FSI

Kennedy Space Center, Florida 32899

\author{
Valter Ferrero \\ Politecnico di Torino \\ Department of Electrical Engineering \\ 10128 Turin, Italy
}

\begin{abstract}
It is well know that free-space laser system performance is limited by atmospheric turbulence. Most theoretical treatments have been described for many years by Kolmogorov's power spectral density model because of its simplicity. Unfortunately, several experiments have been reported recently that show that the Kolmogorov theory is sometimes incomplete to describe atmospheric statistics properly, in particular, in portions of the troposphere and stratosphere. We present a nonKolmogorov power spectrum that uses a generalized exponent instead of constant standard exponent value $11 / 3$, and a generalized amplitude factor instead of constant value 0.033. Using this new spectrum in weak turbulence, we carry out, for a horizontal path, an analysis of long-term beam spread, scintillation index, probability of fade, mean signal-to-noise ratio (SNR), and mean bit error rate (BER) as variation of the spectrum exponent. Our theoretical results show that for alpha values lower than $\alpha=11 / 3$, but not for alpha close to $\alpha=3$, there is a remarkable increase of scintillation and consequently a major penalty on the system performance. However, when alpha assumes a value close to $\alpha=3$ or for alpha values higher than $\alpha=11 / 3$, scintillation decreases, leading to an improvement on the system performance. () 2008 Society of Photo-Optical Instrumentation Engineers. [DOI: 10.1117/1.2870113]
\end{abstract}

Subject terms: atmospheric turbulence; structure function; Kolmogorov spectrum; beam spread; scintillation; fade; signal-to-noise ratio; bit error rate.

Paper 070450R received May 26, 2007; revised manuscript received Sep. 30, 2007; accepted for publication Oct. 3, 2007; published online Feb. 28, 2008. This paper is a revision of a paper presented at the SPIE conference on Free-Space Laser Communication Technologies XIX and Atmospheric Propagation of Electromagnetic Waves, Jan. 2007, San Jose, California. The paper presented there appears (unrefereed) in SPIE Proceedings Vol. 6457.

\section{Introduction}

For a long time, structure function has been modeled according to Kolmogorov's power spectrum of refractive index fluctuations, which is widely accepted and has been applied extensively in studies of optical and radio wave propagation in the atmosphere. However, recent experimental data from space-based stellar scintillation, balloonborne in-situ temperature, and ground-based radar measurements indicate that turbulence in the upper troposphere and stratosphere deviates from predictions of the Kolmogorov model. ${ }^{1-3}$ One circumstance in which the spectrum may not be Kolmogorov is when the atmosphere is extremely stable, for example, in low wind conditions. In such cases, the rate of the energy cascade from larger to smaller scales is reduced and Kolmogorov turbulence is not fully developed. The Kolmogorov model makes several assumptions about the nature of the turbulence. For example, the turbulent fluctuations of the index of refraction are assumed to be homogeneous, or statistically stationary, and isotropic, meaning there is no preferred direction through the turbulence, and there is a specific mathematical form for the power spectral density of the index of refraction fluctua-

0091-3286/2008/\$25.00 ๑ 2008 SPIE tions. However, further development of the turbulent theory of passive scalar transfer has shown that although the Kolmogorov spectrum is generally correct (within the inertial subrange), it constitutes only one part of the more general behavior of passive scalar transfer in a turbulent flow. ${ }^{4}$ In fact, media with Kolmogorovs-type turbulence are sufficiently wide spread, but they are not the only possible ones. Another type of turbulence as wide spread as Kolmogorov's is helical. ${ }^{5,6}$ In brief, it was shown in the early 1960's that the Euler equation has, side by side with energy, another integral of motion: the helicity. Helicity exists practically in all real turbulent media, and it is necessary only to establish in which cases it is important for the solution of radiation transfer problems. Consequently, since radiation affects temperature fluctuations, then in the presence of nonzero helicity and in the case where its flux is a governing parameter, besides spectral properties of the velocity field, passive scalar behavior is also changed. It has also been reported ${ }^{7}$ that some anomaly behavior seems to occur when the atmosphere is extremely stable, because under such conditions the turbulence is no longer homogeneous in three dimensions, since the vertical component is suppressed. It has been shown ${ }^{8}$ that for such 2-D turbulence, coherent vortices can develop that reduce the rate of the 
energy cascade from larger to smaller scales. As a result, Kolmogorov turbulence will not develop. In addition, recent experimental results ${ }^{9}$ have indirectly indicated that near the tropopause, the Kolmogorov model may not completely describe the power spectral density of the turbulence. Therefore, a significant body of evidence that conflicts with the conventional statistical model of turbulence is now available from various measurements of turbulence under a variety of conditions. In addition, anisotropy in stratospheric turbulent inhomogeneities has been experimentally investigated. ${ }^{10-13}$ Also, laboratory investigations have shown that turbulence can be anisotropic, even at Reynolds numbers well past the critical Reynolds number for the flow geometry. Since the tropopause (i.e., the boundary between the troposphere and the stratosphere) is known to have fluid flow properties similar to the shear layers created in the laboratory, these studies cast doubt on the correctness of the conventional assumption of isotropic turbulence through the entire atmosphere. French researchers ${ }^{9}$ have collected data at high altitudes that indicate the existence of anisotropic turbulence and nonKolmogorov behavior, and recent velocity scale size data collected from an aircraft report anisotropic behavior in the tropopause. Flight test studies have shown the presence of stratified layers in the upper atmosphere at altitudes between 12 and $18 \mathrm{~km}$, indicating that conventional models of how turbulence strength smoothly decreases as a function of altitude may be in error. In addition, there have been a number of successful measurement campaigns to quantify the lower atmosphere, where the density and turbulence levels are orders of magnitude higher than the upper atmosphere. From these measurements, assumptions have been made as to the nature of the turbulence, and the techniques used in measuring the lower altitude turbulence have been applied to high altitude measurements. However, turbulence generation and dissipation are fundamentally different at high and low altitudes. Where the lower regions are influenced by the surface of the earth, atmospheric density, and molecular dissipation of energy, the high regions are dominated by large-scale turbulence generation phenomena, strong discontinuity associated with the tropopause, and solar flux. Finally, turbulence spectrum was investigated by Lidar measurements. ${ }^{14}$ The experimental results show the various strata and layers in the vertical turbulence profiles. It is shown that the power-law exponent of the structure function is different from the purely Kolmogorov cases. Thus, it should be taken into account in applications of turbulence sensing, image restoration, free-space optical communications, etc. We must accept de facto that turbulence is still an unsolved problem in classical physics, and the scientific community must persist in doing more simulations, measurements, and experiments. ${ }^{15}$

Stewart McKechnie reported, ${ }^{16}$ "...the time has come to look at the subject of imaging through turbulence much more critically. It might be more constructive to abandon old dogma and replace it with a sounder and more open approach."

It is very important, therefore, to find new models more general than the Kolmogorov spectrum to describe experimental data also in non-Kolmogorov turbulence. In this work, we present a theoretical spectrum model that reduces to one type of Kolmogorov only for a particular case of its exponent: the standard value $11 / 3$. The exponent can assume all the values between the range 3 to 4 . Using this new spectrum, following the same procedure already used by Andrews, Phillips, et al., ${ }^{17,18}$ we have analyzed the impact of the exponent's variation on long-term beam spread, scintillation index, probability of fade, mean signal-to-noise ratio (SNR), and mean bit error rate (BER) for a horizontal path, that is, for constant value of the refractive index structure parameter.

\section{Non-Kolmogorov Spectrum}

The basic power-law spectrum of Kolmogorov is defined by

$\Phi_{n}(\kappa)=0.033 \cdot C_{n}^{2} \cdot \kappa^{-11 / 3}$,

where $C_{n}^{2}$ is the refractive-index structure parameter. The validity of the Kolmogorov spectrum is restricted to the inertial range, although in some analyses it is extended to all spatial wave numbers. Here we examine a more general power spectrum model that describes non-Kolmogorov atmospheric turbulence, in which the power law exponent $11 / 3$ is allowed to deviate somewhat from this value.

One of the key parameters for developing the theory of radiation passage through a turbulent medium is the structure function $D(r)$. The structure function describes the behavior of correlations of turbulent temperature or other passive scalar (aerosol, for example) field fluctuations with distance between two given points separated by a distance $r$. We assume that in an atmosphere exhibiting nonKolmogorov turbulence, the structure function for the index of refraction is given by

$D_{n}(r)=\beta \cdot C_{n}^{2} \cdot r^{\gamma}$,

where $\gamma$ is the power law that reduces to $2 / 3$ in the case of conventional Kolmogorov turbulence. Here, $\beta$ is a constant equal to unity when $\gamma=2 / 3$, but otherwise has units $m^{-\gamma+2 / 3}$. Following same procedure reported in Ref. 17, the corresponding power-law spectrum associated with structure function takes the form (see Appendix in Sec. 9)

$\Phi_{n}(\kappa, \alpha)=A(\alpha) \cdot \widetilde{C}_{n}^{2} \cdot \kappa^{-\alpha}, \quad \kappa>0, \quad 3<\alpha<4$,

where $\alpha=\gamma+3$ is the spectral index or power law, $\widetilde{C}_{n}^{2}$ $=\beta \cdot C_{n}^{2}$ is a generalized structure parameter with units $m^{-\gamma}$, $A(\alpha)$ is defined by

$A(\alpha)=\frac{1}{4 \pi^{2}} \Gamma(\alpha-1) \cos \left(\frac{\alpha \pi}{2}\right), \quad 3<\alpha<4$,

and the symbol $\Gamma(x)$ in the last expression is the gamma function. When $\alpha=11 / 3$, we find that $A(11 / 3)=0.033$, and the generalized power spectrum reduces to the conventional Kolmogorov spectrum in Eq. (1). Also, when the power law approaches the limiting value $\alpha=3$, the function $A(\alpha)$ approaches zero. Consequently, the refractive-index power spectral density vanishes in this limiting case.

\section{Long-Term Beam Spread}

The first important quantity that shows total average beam spot size radius on the receiver lens is the long-term beam 


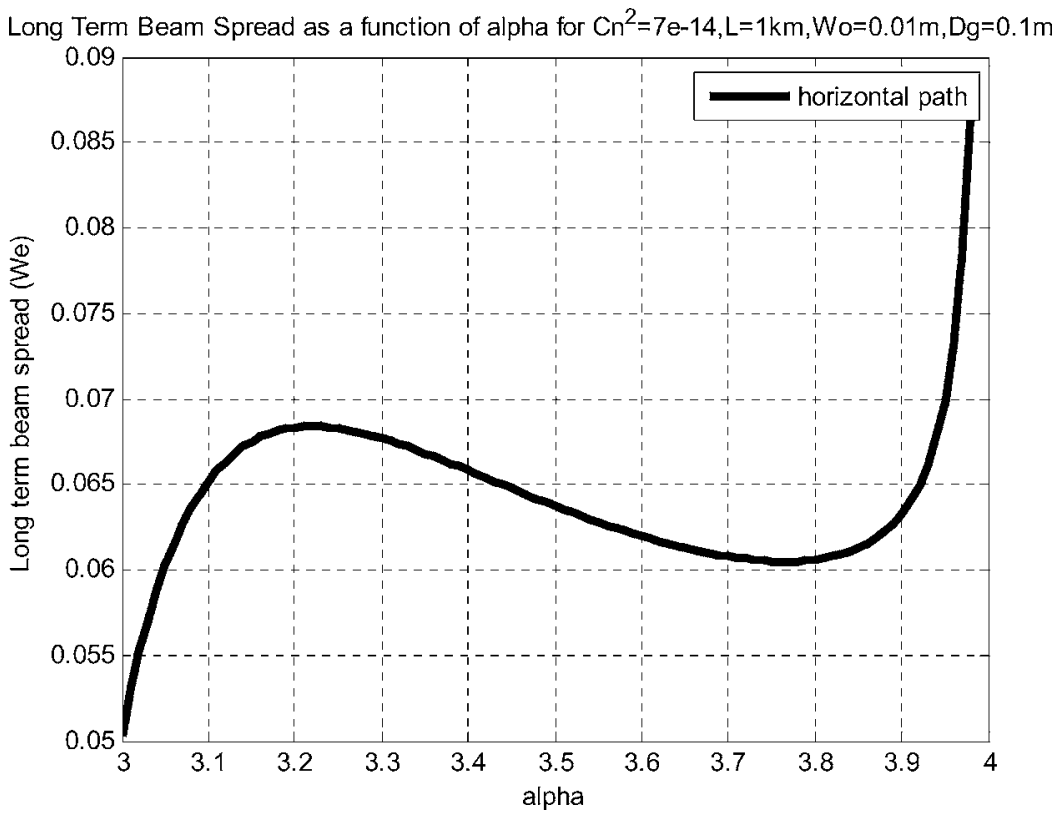

Fig. 1 Long-term beam spread as a function of alpha for horizontal path.

spread. It can be written as the sum of three terms: diffraction-limited beam spreading, beam spreading due to small turbulence scales, and beam wander, which can be described by the variance of the instantaneous center of the beam in the receiver plane.

The analytical form of long-term beam spread for a Gaussian beam wave is ${ }^{17}$

$W_{e}^{2}=\left\langle W_{L T}^{2}(\alpha)\right\rangle=W^{2} \cdot[1+\langle T(\alpha)\rangle]$,

where $W$ is the diffraction-limited spot size radius and $\langle T(\alpha)\rangle$ is the term that includes small-scale beam spreading and beam wander atmospheric effects. To carry out longterm beam spread analysis, we need to calculate the $\langle T(\alpha)\rangle$ term. For a horizontal path, the parameter $C_{n}^{2}$ that appears inside the relation $\widetilde{C}_{n}^{2}=\beta \cdot C_{n}^{2}$ is constant. Following the same formula reported in Ref. 17, but using the nonKolmogorov spectrum in Eq. (3), we carry out

$$
\begin{aligned}
T(\alpha)= & 4 \pi^{2} k^{2} L \cdot \int_{0}^{1} \int_{0}^{\infty} \kappa \cdot \phi_{n}(\alpha, \kappa) \\
& \times\left[1-\exp \left(-\frac{\Lambda L \kappa^{2} \xi^{2}}{k}\right)\right] d \kappa d \xi \\
= & -16 \cdot A(\alpha) \cdot \frac{1}{\alpha-1} \cdot \Gamma\left(1-\frac{\alpha}{2}\right) \cdot \Lambda^{\alpha / 2-1} \cdot \widetilde{\sigma}_{R}^{2}(\alpha),
\end{aligned}
$$

where $\xi=1-z / L \quad(z$ is the propagation distance), $\Lambda$ $=2 L / k W^{2}$, and we have defined a non-Kolmogorov Rytov variance

$\widetilde{\sigma}_{R}^{2}(\alpha)=1.23 \cdot \widetilde{C}_{n}^{2} \cdot k^{3-\alpha / 2} \cdot L^{\alpha / 2}$.

It is interesting to observe that for $\alpha=11 / 3$, we obtain the particular case of the Kolmogorov spectrum already reported in Ref. 17.
At this point, we plot in Fig. 1 the long-term beam spread as a function of $\alpha$ for a particular case, taking:

$L=1 \mathrm{~km} ; \quad \tilde{C}_{n}^{2}=7 \cdot 10^{-14} \mathrm{~m}^{-\alpha+3} ;$

$\lambda=1.55 \mu \mathrm{m} ; \quad W_{0}=0.01 \mathrm{~m}$.

We deduce from Fig. 1 that if alpha decreases from $\alpha$ $=11 / 3$ (excluding alpha values close to 3 ), then the longterm beam spread $W_{e}$ increases up to a maximum value. At this point, the curve changes its slope, because the term $A(\alpha)$ assumes very low values. In addition, it is shown that if alpha increases from $\alpha=11 / 3$, then the long-term beam spread $W_{e}$ decreases down to a minimum value. At this point, the curve changes its slope, because the term $\Gamma(1$ $-\alpha / 2$ ) assumes high values close to its asymptote as $\alpha$ $\rightarrow 4$. The obvious physical interpretation of alpha approaching 3 is that turbulence tends to vanish. On the other extreme, the physical interpretation of alpha approaching 4 is that phase effects dominate in the form of random tilts, which generate beam wander.

Finally, it is interesting to observe that recently in literature there have appeared several works concerning other beam types and their advantages over the pure Gaussian beam type. ${ }^{19,20}$ Unfortunately, we do not yet know how our non-Kolmogorov spectrum would affect such cases. However, we presume that different beam types probably will present the same advantages over the pure Gaussian beam like those they present by using the Kolmogorov spectrum. For example, beam spreading for higher-order Gaussian beams, by using our non-Kolmogorov spectrum, probably will be less than that of a lowest-order Gaussian beam. That is, because the higher-order mode structure places more energy of the beam away from the beam axis, the incremen- 
tal spreading due to turbulence should be less also in nonKolmogorov turbulence. A similar result will most likely be obtained also for annular beams.

\section{Scintillation}

Another important parameter that is necessary to calculate the system performance is the scintillation index. In our analysis, we include aperture averaging effects of the receiver aperture, so we carry out the flux variance in the plane of the detector at a short distance behind the collecting lens of diameter $D_{G}$. Finally, in our analysis we assume that beam-wander-induced scintillation is negligible, which is true when we consider either a plane wave or a spherical wave, but the situation may be different for focused beams. $^{17}$

\subsection{Horizontal Path: Plane Wave and Spherical Wave Models}

Following the same procedure as discussed in Ref. 17 for the standard Kolmogorov spectrum, but this time using a non-Kolmogorov spectrum, our analysis for the plane wave model leads to

$$
\begin{aligned}
\sigma_{I_{\text {_plane }}}^{2}\left(\alpha, D_{G}\right)= & 8 \pi^{2} \cdot k^{2} \cdot L \cdot \operatorname{Re}\left\{\int_{0}^{1} \int_{0}^{\infty} \kappa \cdot \Phi_{n}(\kappa, \alpha)\right. \\
& \cdot \exp \left(-\frac{D_{G}^{2} \cdot \kappa^{2}}{16}\right) \\
& \left.\cdot\left[1-\exp \left(-j \frac{L \cdot \kappa^{2} \cdot \xi}{k}\right)\right] d \kappa d \xi\right\} \\
= & 6.5 \cdot \pi^{2} \cdot A(\alpha) \cdot \widetilde{\sigma}_{R}^{2}(\alpha) \\
& \cdot \Gamma\left(1-\frac{\alpha}{2}\right) \cdot \frac{1}{\alpha} \cdot\left\{\frac{\alpha}{2} \cdot\left(\frac{k \cdot D_{G}^{2}}{16 \cdot L}\right)^{\alpha / 2-1}\right. \\
& -\left[\left(\frac{k \cdot D_{G}^{2}}{16 \cdot L}\right)^{2}+1\right] \alpha / 4 \\
& \left.\cdot \sin \left[\frac{\alpha}{2} \cdot \operatorname{arctg}\left(\frac{16 \cdot L}{k \cdot D_{G}^{2}}\right)\right]\right\} .
\end{aligned}
$$

Our analysis for the spherical wave model leads to

$$
\begin{aligned}
\sigma_{I_{-} \text {spherical }}^{2}\left(\alpha, D_{G}\right) & 8 \pi^{2} \cdot k^{2} \cdot L \cdot \operatorname{Re}\left\{\int_{0}^{1} \int_{0}^{\infty} \kappa \cdot \Phi_{n}(\kappa, \alpha)\right. \\
\cdot & \exp \left(-\frac{D_{G}^{2} \cdot \kappa^{2} \cdot \xi^{2}}{16}\right) \\
\cdot & {\left.\left[1-\exp \left(-j \frac{L \cdot \kappa^{2} \cdot \xi \cdot(1-\xi)}{k}\right)\right] d \kappa d \xi\right\} } \\
= & 4 \pi^{2} \cdot k^{2} \cdot L \cdot A(\alpha) \cdot \widetilde{C}_{n}^{2} \cdot\left(\frac{16}{D_{G}^{2}}\right)^{1-\alpha / 2} \\
\cdot & \Gamma\left(1-\frac{\alpha}{2}\right) \cdot\left\{\frac{1}{\alpha-1}-\operatorname{Re}\left[\left(j \frac{16 L}{k D_{G}^{2}}\right)^{\alpha / 2-1}\right.\right.
\end{aligned}
$$

$$
\left.\left.\cdot \frac{2}{\alpha} \cdot{ }_{2} F_{1}\left(1-\frac{\alpha}{2}, \frac{\alpha}{2} ; 1+\frac{\alpha}{2} ; 1+j \frac{k D_{G}^{2}}{16 L}\right)\right]\right\},
$$

where ${ }_{2} F_{1}(a, b ; c ; z)$ is the hypergeometric function ${ }^{17}$ given by

${ }_{2} F_{1}(a, b ; c ; z)=\sum_{0}^{\infty} \frac{(a)_{n} \cdot(b)_{n}}{(c)_{n}} \cdot \frac{z^{n}}{n !}, \quad|z|<\infty$.

At this point, we can plot both $\sigma_{I_{\text {_plane }}}^{2}\left(\alpha, D_{G}\right)$ and $\sigma_{I_{-} \text {spherical }}^{2}\left(\alpha, D_{G}\right)$ as a function of alpha for a particular horizontal case. We take

$L=1 \mathrm{~km} ; \quad \tilde{C}_{n}^{2}=7 \cdot 10^{-14} \mathrm{~m}^{-\alpha+3} ;$

$\lambda=1.55 \mu \mathrm{m} ; \quad D_{G}=0.1 \mathrm{~m}$.

The results for both the plane wave and spherical wave are shown in Fig. 2. We deduce from Fig. 2 that for alpha values lower than Kolmogorov value $\alpha=11 / 3$, excluding alpha values close to 3 , there is an increase of scintillation both for the spherical wave model and the plane wave model, but for the spherical wave model it is more pronounced. Consequently, scintillation in this case leads to a larger penalty on system performance. We deduce also that there are two maximum values of scintillation respectively for alpha values close to 3.3 for the plane wave model and close to 3.2 for the spherical wave model. At these points, the curves change their slopes because the term $A(\alpha)$ begins to decrease to zero. In addition for alpha values higher than $\alpha=11 / 3$, scintillation slightly decreases for both the plane wave model and spherical wave model, and consequently it will lead to a slight gain in system performance.

The physical interpretation of alpha approaching 4 is that the power spectrum contains fewer eddies of high wave numbers; therefore, scintillation effects are reduced.

\section{Probability of Fade}

Given a PDF model for irradiance fluctuations $p_{I}(I)$, the probability of fade describes the percentage of time the irradiance of the received signal is below some prescribed threshold value $I_{T}$. Hence, the probability of fade as a function of threshold level is defined by the cumulative probability $^{17}$

$p_{I}\left(I<I_{T}\right)=\int_{0}^{I_{T}} p_{I}(I) d I$.

The PDF most often used under weak irradiance fluctuations is the lognormal model, and the resulting probability of fade leads to

$p_{I}\left(I<I_{T}\right)=\frac{1}{2} \cdot\left\{1+\operatorname{erf}\left[\frac{\frac{1}{2} \cdot \sigma_{I}^{2}\left(\alpha, D_{G}\right)-0.23 \cdot F_{T}}{\sqrt{2} \cdot \sigma_{I}\left(\alpha, D_{G}\right)}\right]\right\}$,

where $\operatorname{erf}(x)$ is the error function. In arriving at this expression, we have introduced the fade threshold parameter 


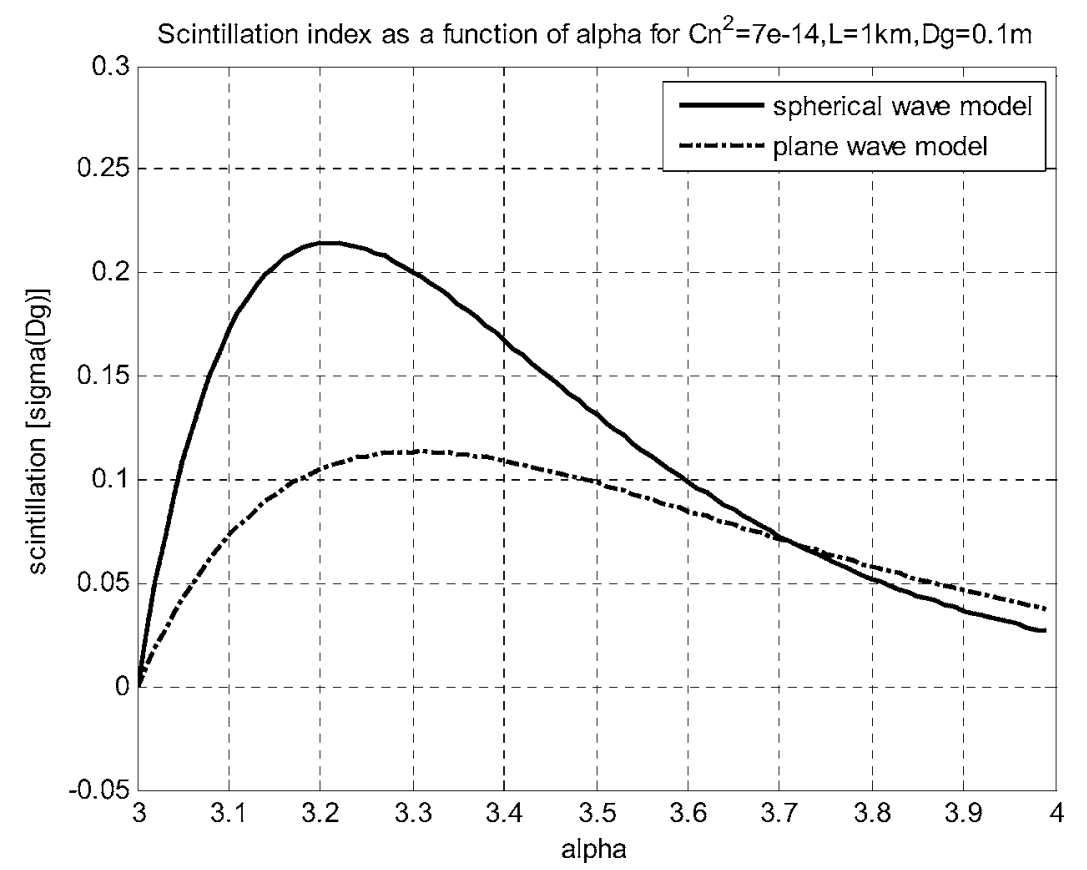

Fig. 2 Scintillation index as a function of alpha for horizontal path using the spherical and plane wave models.

$F_{T}=10 \cdot \log _{10}\left(\frac{\langle I\rangle}{I_{T}}\right)[\mathrm{dB}]$.

The fade parameter $F_{T}$, given in decibels $[\mathrm{dB}]$, represents the decibel level below the on-axis mean irradiance that the threshold $I_{T}$ is set.

\subsection{Horizontal Link: Plane Wave Model and Spherical Wave Model}

Using the scintillation index for the plane wave model [Eq. (8)] and for the spherical wave model [Eq. (9)] in Eq. (12), we calculate the probability of fade as a function of alpha for a fixed fade threshold parameter for a particular horizontal case, in which

$$
\begin{aligned}
& L=1 \mathrm{~km} ; \quad \tilde{C}_{n}^{2}=7 \cdot 10^{-14} \mathrm{~m}^{-\alpha+3} ; \\
& \lambda=1.55 \mu \mathrm{m} ; \quad D_{G}=0.1 \mathrm{~m}, \quad F_{T}=6 \mathrm{~dB} .
\end{aligned}
$$

The plots are shown in Fig. 3 for both the plane wave and spherical wave models.

It is clear now that alpha variation has a serious impact on the probability of fade for both spherical and plane wave models; in particular, lower alpha values lead to a penalty on the fade performance (excluding alpha values close to 3). In addition, we can observe that the spherical wave model predicts a higher probability of fade than the plane wave model for alpha values lower than the alpha value intersection point (around $\alpha=3.72$ ). For alpha values higher than the alpha value of intersection point, the situation is the opposite. Also for this diagram, there is a maximum point where the curves change their slope, because the scintillation begins to decrease to zero.
Under the same conditions, we plot the probability of fade as a function of fade threshold parameter for several alpha values. Both plane wave and spherical wave cases are illustrated respectively in Figs. 4 and 5.

\section{Mean Signal-to-Noise Ratio}

In this section, we examine the mean signal-to-noise ratio in the presence of atmospheric turbulence using a nonKolmogorov power spectrum. The received irradiance over long measurement intervals must be treated like a random variable because of the turbulence. Based on Refs. 17 and 18 , the mean signal-to-noise ratio (SNR) at the output of the detector in the case of a shot-noise limited system assumes the form

$\langle\mathrm{SNR}\rangle=\frac{\mathrm{SNR}_{0}}{\left[1+\sigma_{I}^{2}\left(\alpha, D_{G}\right) \cdot \mathrm{SNR}_{0}^{2}\right]^{1 / 2}}$,

where $\mathrm{SNR}_{0}$ is the signal-to-noise ratio in the absence of turbulence.

\subsection{Horizontal Link: Plane Wave Model}

We plot in decibel units the mean signal-to-noise ratio $\langle\mathrm{SNR}\rangle$ as a function of signal-to-noise ratio without turbulence $\mathrm{SNR}_{0}$ for several alpha values, using the plane wave model for scintillation. We take the following parameters

$L=1 \mathrm{~km} ; \quad \tilde{C}_{n}^{2}=7 \cdot 10^{-14} \mathrm{~m}^{-\alpha+3} ;$

$\lambda=1.55 \mu \mathrm{m} ; \quad D_{G}=0.1 \mathrm{~m}$.

The plot, shown in Fig. 6, illustrates the impact of the alpha variation on the $\langle\mathrm{SNR}\rangle$ performance. Again, when alpha is lower than $\alpha=11 / 3$, excluding alpha values close to 3 , 


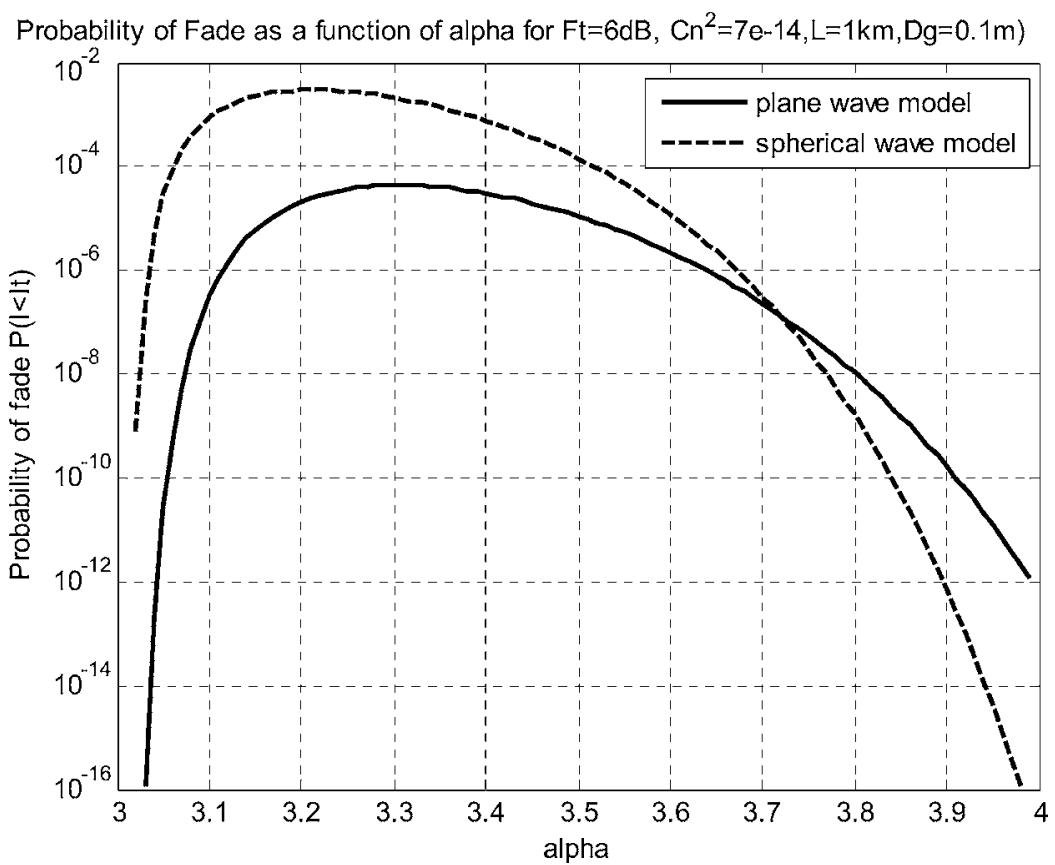

Fig. 3 Probability of fade as a function of alpha using the log-normal PDF and two different models for the scintillation index: plane and spherical wave models.

there is a translation of the curves toward lower values, or, in other words, there is a penalty on the system performance. For alpha values higher than $\alpha=11 / 3$, there is a gain on the system performance with respect to the case of Kolmogorov $\alpha=11 / 3$. Finally, there is also a gain on system performance with respect to Kolmogorov $\alpha=11 / 3$ when alpha assumes values very close to $\alpha=3$, because the amplitude factor $A(\alpha)$ assumes very low values and conse- quently the scintillation reported before in Fig. 2 approaches zero.

\section{Mean Bit Error Rate}

In the presence of optical turbulence, the probability of error is considered a conditional probability that must be averaged over the PDF of the random signal to determine

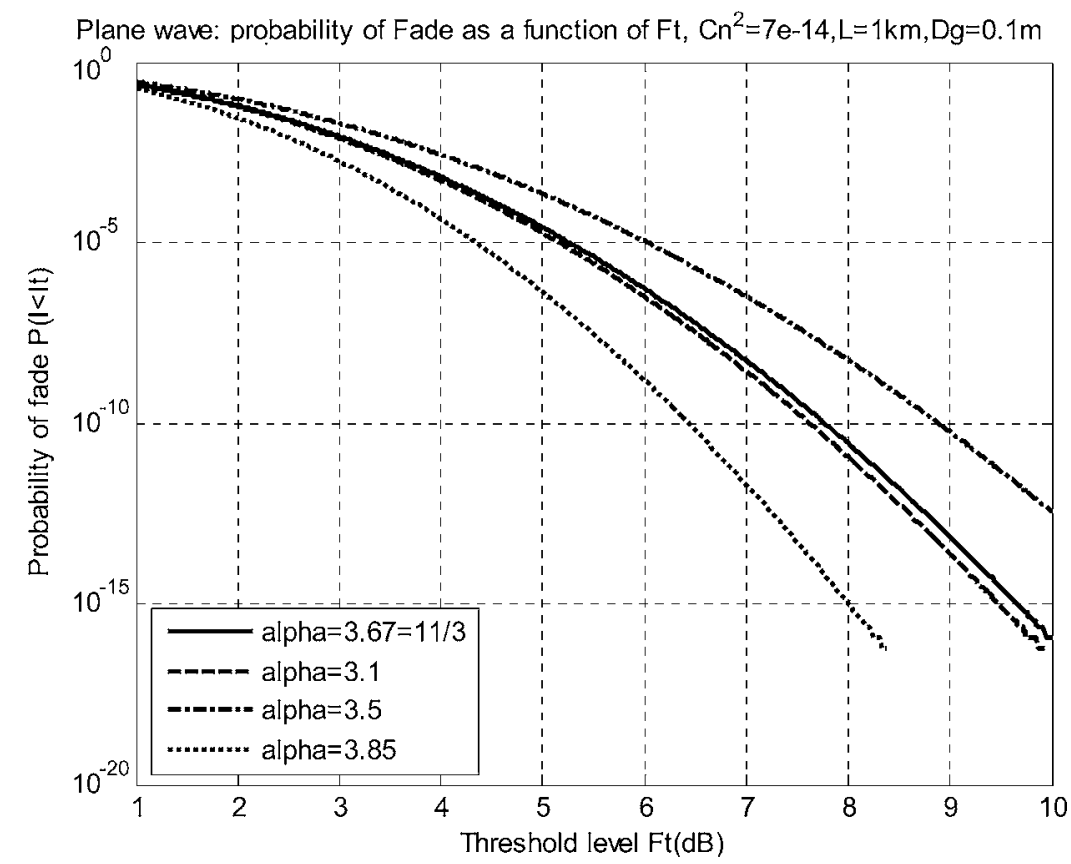

Fig. 4 Probability of fade as a function of threshold level for several values of alpha using the plane wave scintillation index. 
Toselli et al.: Free space optical system performance...

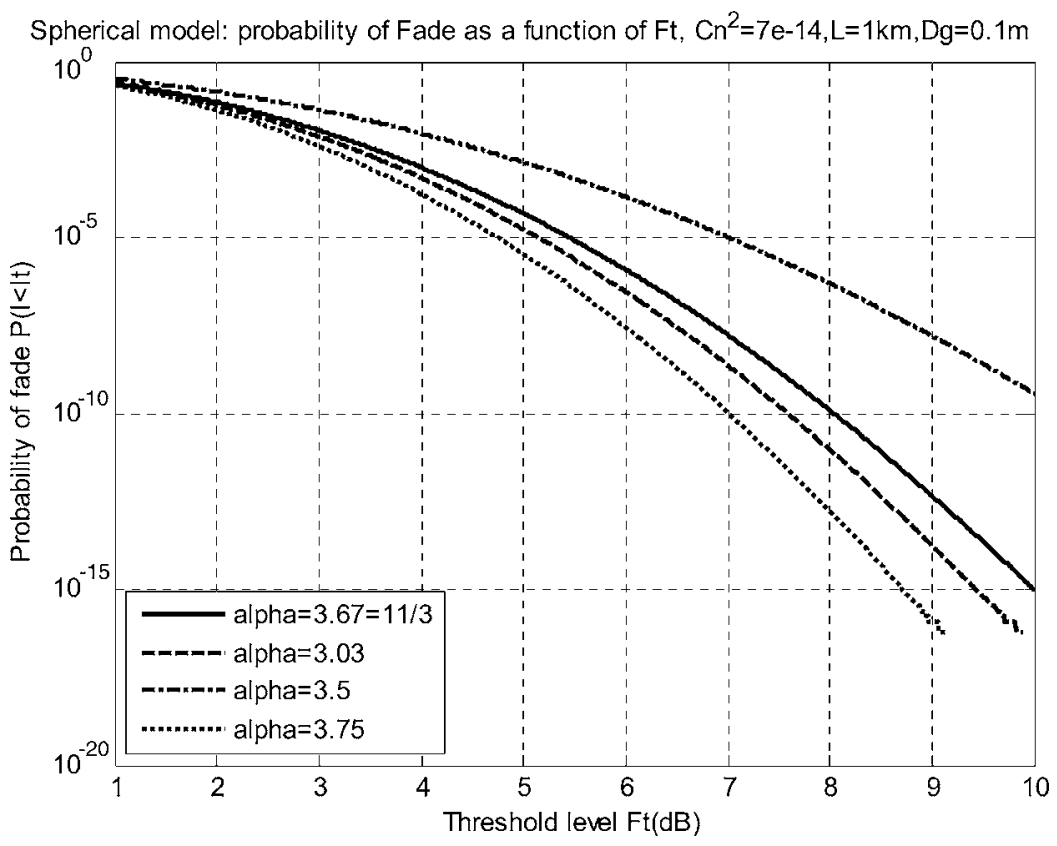

Fig. 5 Probability of fade as a function of threshold level for several values of alpha using the spherical wave scintillation index.

the unconditional mean bit error rate (BER). In terms of a normalized signal with unit mean, this leads to the expression $^{17,18}$

$\operatorname{Pr}(E)=\langle\mathrm{BER}\rangle=\frac{1}{2} \cdot \int_{0}^{\infty} p_{I}(u) \cdot \operatorname{erfc}\left(\frac{\langle\mathrm{SNR}\rangle \cdot u}{2 \cdot \sqrt{2}}\right) d u$,

where $p_{I}(u)$ is taken to be the log normal distribution with unit mean, i.e.,

$$
p_{I}(u)=\frac{1}{u \cdot \sigma_{I}\left(D_{G}, \alpha\right) \cdot \sqrt{2 \cdot \pi}}
$$

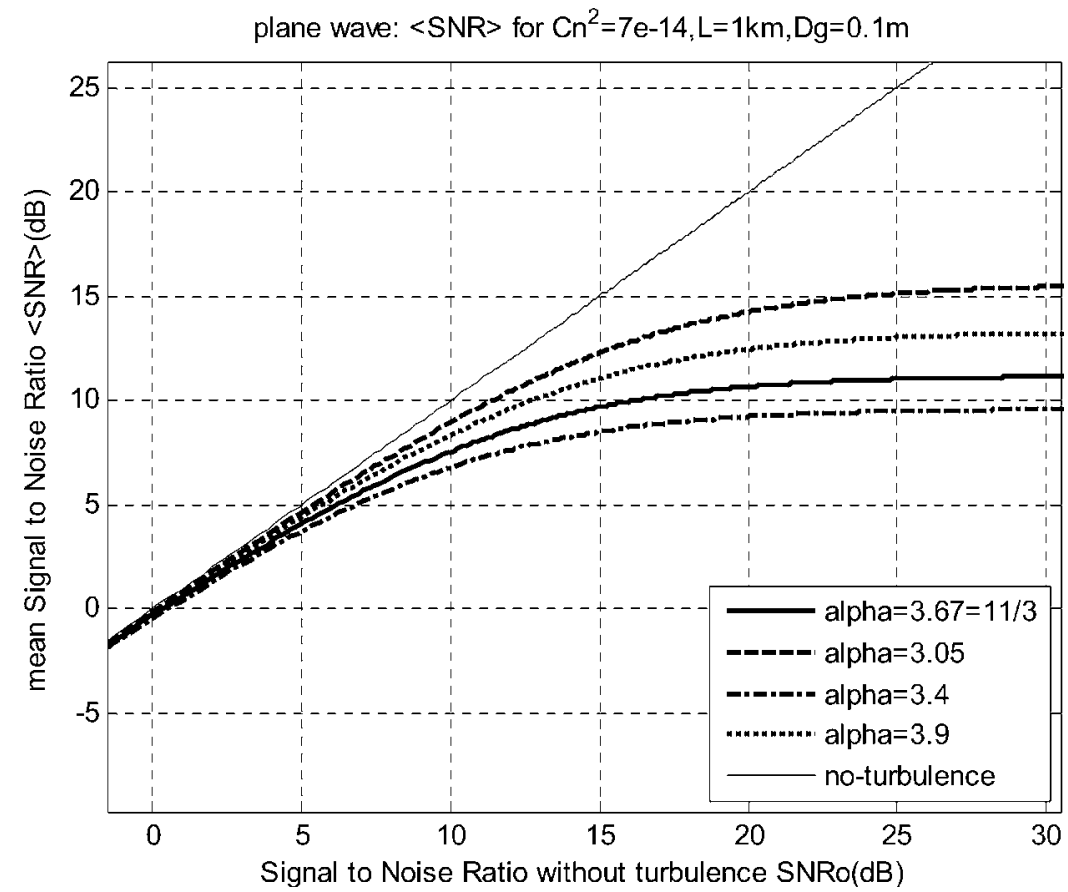

Fig. 6 Mean signal-to-noise ratio as a function of signal-to-noise ratio without turbulence for several alpha values, using the plane wave model for scintillation. 


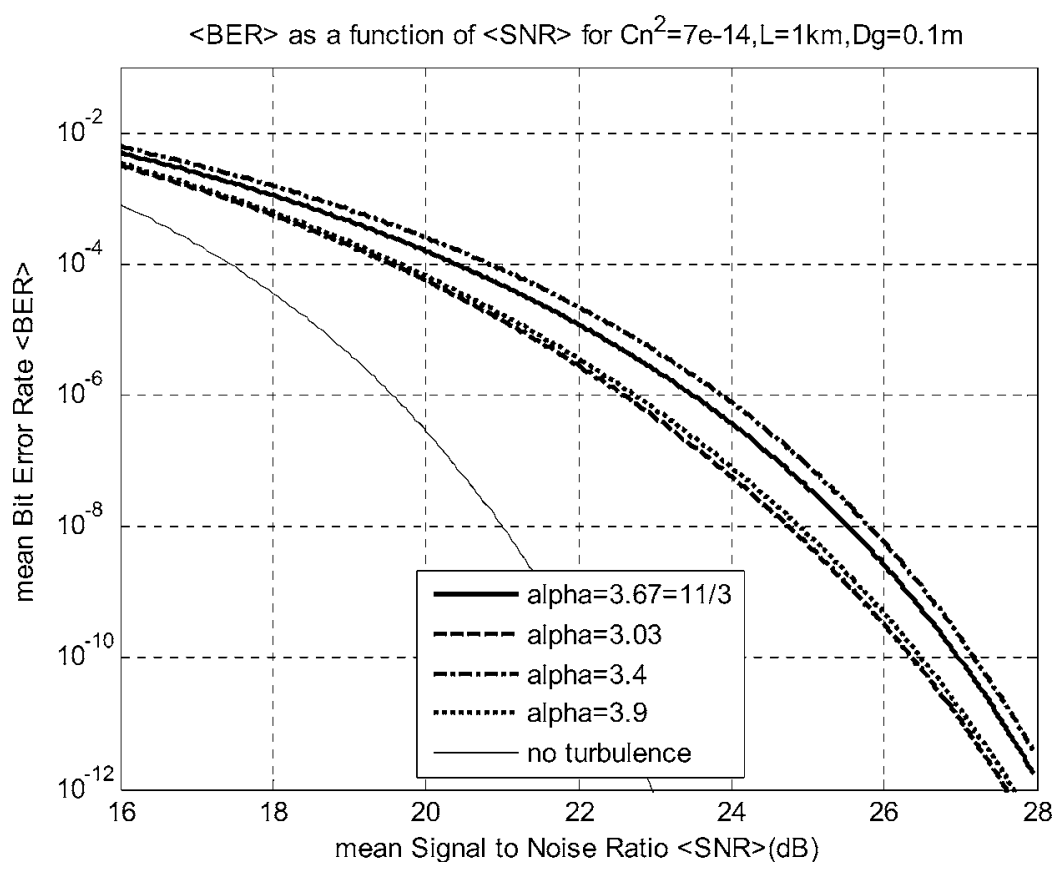

Fig. 7 Mean bit error rate as a function of mean signal-to-noise ratio for several alpha values, using the plane wave model for scintillation.

\subsection{Horizontal Link: Plane Wave Model}

We plot the mean bit error rate as a function of $\langle\mathrm{SNR}\rangle$ for several alpha values using the plane wave model for scintillation. We take the same parameters

$L=1 \mathrm{~km} ; \quad \widetilde{C}_{n}^{2}=7 \cdot 10^{-14} \mathrm{~m}^{-\alpha+3} ;$

$\lambda=1.55 \mu \mathrm{m} ; \quad D_{G}=0.1 \mathrm{~m}$.

The plot is shown in Fig. 7. It shows the impact of the alpha variation on $\langle\mathrm{BER}\rangle$ performance. Also in this analysis, when alpha is lower than $\alpha=11 / 3$, excluding alpha values close to 3 , there is a penalty, but for alpha higher than $\alpha=11 / 3$, there is an improvement on the system performance. However, when alpha assumes values close to $\alpha=3$, there is a gain on the $\langle\mathrm{BER}\rangle$ performance with respect to $\langle\mathrm{BER}\rangle$ value corresponding to $\alpha=11 / 3$, because the scintillation approaches zero.

\section{Discussion}

We introduce a non-Kolmogorov power spectrum that uses both a generalized exponent and a generalized amplitude factor instead of a constant standard exponent value $\alpha$ $=11 / 3$ and a constant amplitude factor 0.033 associated with the conventional Kolmogorov spectrum. This nonKolmogorov spectrum is developed from a generalized structure function. It is shown, for a horizontal link, the long-term beam spread, scintillation, probability of fade, mean SNR, and mean BER as variations depending on the alpha exponent, lead to results somewhat different than those obtained with the standard value of Kolmogorov $\alpha$ $=11 / 3$.

For horizontal links, it is shown that for alpha values lower than $\alpha=11 / 3$, but not for alpha close to $\alpha=3$, there is a remarkable increase of scintillation and consequently a major penalty on system performance. However, when alpha assumes a value close to $\alpha=3$, the amplitude factor $A(\alpha)$ assumes a very low value and consequently the longterm beam spread and scintillation decrease, leading to an improvement on the system performance. Finally, also for higher alpha values than $\alpha=11 / 3$, the scintillation decreases and consequently improves system performance.

\section{Appendix}

The power spectrum and structure function are related by ${ }^{17}$

$\Phi_{n}(\kappa, \alpha)=\frac{1}{4 \pi^{2} \kappa^{2}} \int_{0}^{\infty} \frac{\sin (\kappa r)}{\kappa r} \cdot \frac{\partial}{\partial r}\left(r^{2} \frac{\partial D_{n}(r, \beta)}{\partial r}\right) d r$,

where the refractive-index structure function $D_{n}(r, \beta)$ is given by Eq. (2).

Using the integral formula ${ }^{17}$

$\int_{0}^{\infty} x^{\alpha} \sin (x) d x=2^{\alpha} \sqrt{\pi} \frac{\Gamma\left(\frac{\alpha}{2}+1\right)}{\Gamma\left(\frac{1}{2}-\frac{\alpha}{2}\right)}$,

we obtain the final result

$\Phi_{n}(\kappa, \alpha)=A(\alpha) \cdot \widetilde{C}_{n}^{2} \cdot \kappa^{-\alpha}$,

where 


$$
\begin{aligned}
A(\alpha) & =\frac{(\alpha-3) \cdot(\alpha-2) \cdot 2^{\alpha-4}}{4 \pi^{3 / 2}} \cdot \frac{\Gamma\left(\frac{\alpha}{2}-1\right)}{\Gamma\left(\frac{5}{2}-\frac{\alpha}{2}\right)} \\
& =\frac{1}{4 \pi^{2}} \Gamma(\alpha-1) \cos \left(\frac{\alpha \pi}{2}\right) .
\end{aligned}
$$

\section{References}

1. M. S. Be1enkii, S. J. Karis, J. M. Brown II, and R. Q. Fugate, "Experimental study of the effect of non Kolmogorov stratospheric turbulence on star image motion," Proc. SPIE 3126, 113-123 (1997).

2. B. E. Stribling, B. M. Welsh, and M. C. Roggemann, "Optical propagation in non Kolmogorov atmospheric turbulence," Proc. SPIE 2471, 181-196 (1995).

3. D. T. Kyrazis, J. Wissler, D. D.B. Keating, A. J. Preble, and K. P. Bishop, "Measurement of optical turbulence in the upper troposphere and lower stratosphere," Proc. SPIE 2120, 43-55 (1994).

4. E. Golbraikh and N. S. Kopeika, "Behavior of structure function of refraction coefficients in different turbulent fields," Appl. Opt. 43(33), 6151-6156 (2004)

5. A. Zilberman, E. Golbraikh, and N. S. Kopeika, "Validity of Kolmogorov turbulence at higher elevations," Proc. SPIE 5160, 397-405 (2004).

6. A. Brissaud, U. Frish, J. Leorat, M. Lesieur, and A. Masure, "Helicity cascades in fully developed isotropic turbulence," Phys. Fluids $\mathbf{1 6}$ 1366-1367 (1973).

7. D. Dayton, B. Pierson, and B. Spielbusch, "Atmospheric structure function measurements with a Shack-Hartmann wave front sensor," Opt. Lett. 17(24), 1737-1739 (1992).

8. J. McWilliam, "A demonstration of the suppression of turbulent cascades by coherent vortices in two-dimensional turbulence," Phys. Fluids A A2, 547 (1990).

9. L. J. Otten, L. C. Roggemann, B. Al Jones, J. Lane, and D. G. Black, "High bandwidth atmospheric turbulence data collection platform," Proc. SPIE 3866, 23-32 (1999).

10. A. I. Kon, "Qualitative theory of amplitude and phase fluctuations in a medium with anisotropic turbulent irregularities," Wave Random Media 4, 297-305 (1994).

11. M. S. Belen'kii, S. J. Karis, and C. L. Osmon, "Experimental evidence of the effects of non Kolmogorov turbulence and anisotropy of turbulence," Proc. SPIE 3749, 50-57 (1999).

12. R. R. Beland, "Some aspects of propagation through weak isotropic non Kolmogorov turbulence," Proc. SPIE 2375, 6-16 (1995).

13. M. S. Belenkiia, J. D. Barchers, S. J. Karis, C. L. Osmon, J. M. Brown II, and R. Q. Fugate, "Preliminary experimental evidence of anisotropy of turbulence and the effect of non Kolmogorov turbulence on wavefront tilt statistics," Proc. SPIE 3762, 396-406 (1999)

14. A. Zilberman, E. Golbraikh, and N. S. Kopeika, "Lidar studies of aerosol and non Kolmogorov turbulence in the Mediterranean troposphere," Proc. SPIE 5987, 598702 (2005).

15. G. Falkovich and K. R. Sreenivasan, "Lessons from hydrodynamic turbulence," Phys. Today, 43-49 (Apr. 2006).

16. T. Stewart McKechnie, "General formulation for light propagation and imaging through atmospheric turbulence, and naturally derived turbulence measurement procedures," Proc. SPIE 4976, 34-46 (2003).

17. L. C. Andrews and R. L. Phillips, Laser Beam Propagation through Random Media, 2nd ed., SPIE Press, Bellingham, WA (2005).

18. L. C. Andrews, R. L. Phillips, and C. Y. Hopen, Laser Beam Scintillation with Applications, SPIE Press, Bellingham, WA (2001).

19. H. T. Eyyuboglu and Y. Baykal, "Scintillations of cos-Gaussian and annular beams," J. Opt. Soc. Am. A 24(1), 156-162 (2007).

20. H. T. Eyyuboglu and Y. Baykal, "Scintillations characteristics of cosh-Gaussian beams," Appl. Opt. 46(7), 1099-1106 (2007).

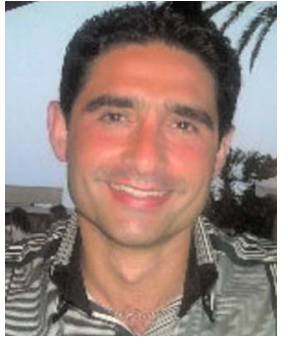

Italo Toselli is working toward a PhD degree in electrical engineering from Politecnico di Torino, Italy. He received a MSc degree in electrical engineering from Università La Sapienza in Rome, and a postgraduated master's in information technology from Cefriel-Politecnico di Milano in Milan, both in 2002. He was an Italian Navy officer from October 2002 to December 2003. Since January 2006, he has been a visiting student at the University of Central Florida (UCF), where he is doing research on free-space optical propagation through atmospheric turbulence.

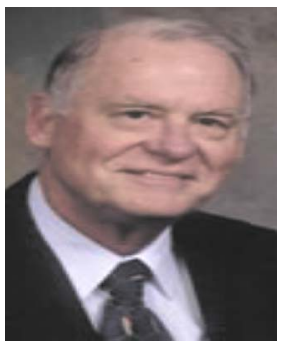

Larry C. Andrews is a professor of mathematics at the University of Central Florida and an associate member of the College of Optics/CREOL. He is also an associate member of the Florida Space Institute. Previously, he held a faculty position at TriState University and was a staff mathematician with the Magnavox Company, antisubmarine warfare operation. He received a doctoral degree in theoretical mechanics in 1970 from Michigan State University. He has been an active researcher in optical wave propagation through random media for more than 25 years, and is the author or coauthor of ten textbooks on topics of differential equations, boundary value problems, special functions, integral transforms, wave propagation through random media, and mathematical techniques for engineers. Along with wave propagation through random media, his research interests include special functions, random variables, atmospheric turbulence, and signal processing.

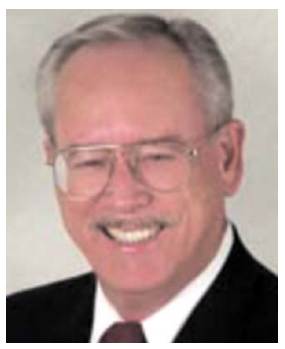

Ronald L. Phillips is director of the Florida Space Institute and a professor in the Department of Electrical and Computer Engineering at the University of Central Florida. $\mathrm{He}$ is also a member of the Department of Mathematics and an associate member of the College of Optics/CREOL. He has held positions on the faculties at Arizona State University and the University of California, San Diego. He received a doctoral degree in electrical engineering in 1970 from Arizona State University. He has been an active researcher in wave propagation through random media for more than 28 years. He was awarded a Senior NATO Postdoctoral Fellowship in 1977, and the American Society for Engineering Education 1983 Medal for outstanding contributions in research. He has coauthored two textbooks on wave propagation through random media and mathematical techniques for engineers. In addition to optical wave propagation, his research interests include optical communications and imaging through atmospheric turbulence.

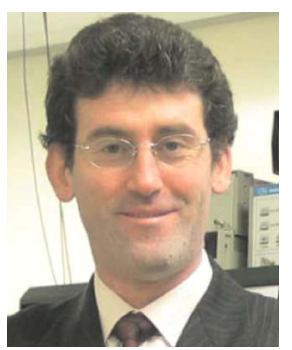

Valter Ferrero received the Laurea in Ingegneria Elettronica (summa cum laude) from Politecnico di Torino, Turin, Italy, in 1994. In 1994 he collaborated with Politecnico di Torino, working on optical coherent systems. From 1995 to 1996, he was with General Electric Company-Marconi (Genova, Italy), where he worked on the design and implementation of Erbium-doped fiber optical amplifiers. He worked also on the design of optical interfaces for Synchronous Digital Hierarchy systems STM4 and STM16. In 1997, he was in charge of the Politecnico di Torino electrical department optical laboratory. In February 2001, he became an assistant professor. He is in the optical communication group in Politecnico di Torino, supervising laboratory conduction, and is involved on several research topics related to optical communications. He is currently involved mainly in optical coherent communications and free-space communications. 